\title{
Etude morphogénétique de la couronne de clones d'Hevea brasiliensis résistant et sensible à la casse au vent $\left(^{*}\right)$
}

\author{
J.P. HOFMANN \\ I.N.R.A., Laboratoire d'Amélioration des Plantes \\ U.P.S., Bâtiment 360, 91405 Orsay-Cedex
}

\begin{abstract}
Résumé
La relation existant chez Hevea brasiliensis entre la forme de la comonn: ai la sensibilité ou la résistance à la casse au vent peut ètre utilisée pour rechercher des criteres de sélection précoce pour ce caractère. Une étude descriptive de la croissance d’un clone résistant (PB 5/51) et d'un clone sensible (RRIM 600) a été effectuée. Un ensemble de variables qui représentent l'édilication et les caractéristiques des axes primaires, secondaites et tertiares, et le fonctionnement des bourgeons à lorigine des ramilications, a été clélini. Les différences entre les 2 clones concernent surtout l'arrêt de la croissance de l'axe primaire, la régularité de la ramification, la longueur, le nombre et la mortalité des branches, leurs angles d'insertion. La variabilité intraclonale est plus forte pour le clone sensible RRIM 600 pour la quasi totalité des caractères étudiés. Le fait de disposer de variables aisément mesurables et présentant des différences entre clone résistant et sensible est une première étape indispensable vers la définition de critères de sélection précoce directement utilisables.
\end{abstract}

\section{Introduction}

Un des probièmes rencontrés dans les plantations d'Hevea brasiliensis (Mïll.-Arg.) est la sensibilité au vent de cet arbre. Ceci se traouit par la casse du trone ef des branches lors de tornades (fig. 1). Il existe une variabilité génétique pour ce caractère qui a pu être mise en évidence dans de très nombreuses expériences de comparaison de clones. Ces différences de sensibilité à la casse sont associées depuis longtemps à différents types de ramification et de formes de couronne (OsTendorf, 1931; Yoon, 1967 ; Wicherley, 1969 ; Anon, 1974 ; Combe \& Duplessix, 1974 ; Hofmann, 1981). Les différents auteurs s'accordent sur les caractéristiques des clones sensibles et résistants :

- les clones sensibles ont des branches maitresses d'un diamètre important dont l'angle d'insertion sur le trone est faible; la forme de la couronne est celle d'un éventail ;

(*) Cet article est un condensé de la thèse de docteur ès-sciences naturelles "Recherche de critères de sélection pour la résistance à la caisse au vent chez Hevea brasiliensis : étude morphogénétique de la couronne et modèle de simulation de la croissance » soutenue le 6 juillet 1981 par l'auteur à l'Université de Paris-Sud (Centre scientifique d'Orsay). 

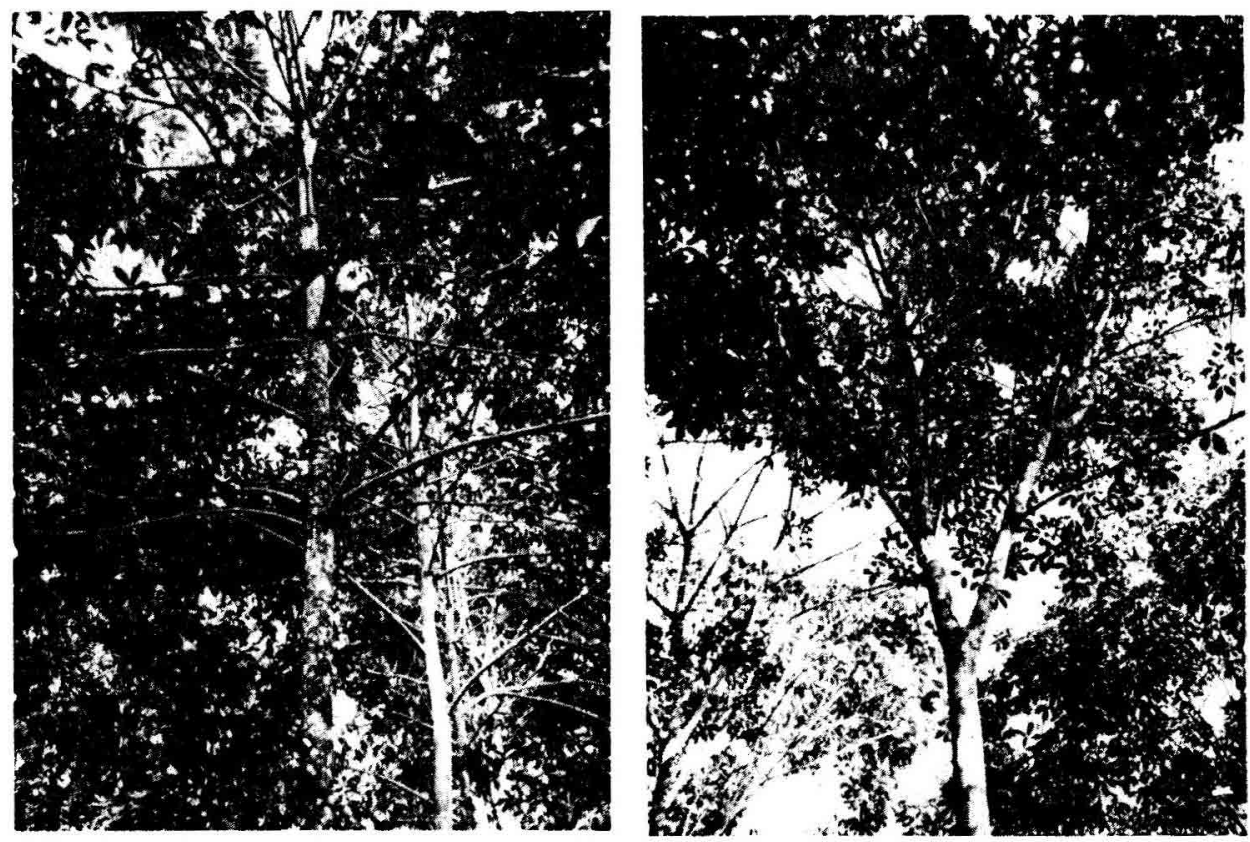

FIG. 1

Formes de couronne sensible et résistante.

Wind resistant and susceptible crownshape.

- Ies clones résistants présentent un tronc droit sans fourches avec des branches fines dont l'angle d'insertion sur le tronc est plus ouvert; la forme de la couronne peut être assimilée à celle d'un cône pointe en haut.

Pour pouvoir prendre en compte dans la sélection le caractère de résistance à la casse au vent, on a cherché à définir des critères de sélection précoce. Deux approches complémentaires ont été utilisées :

- l'étude de paramètres de qualité du bois : densité du bois, contraintes de croissance, bois de tension, résistance à la flexion (Nicolas \& HofmanN, 1977 ; Nicolas et al., 1979);

\section{- l'étude de la morphogénèse de la couronne.}

Dans la deuxième approche, qui est abordée dans cet article. la premièrc étape consistait à définir et à quantifier des paramètres de la morphogénèse susceptibles de représenter les différences entre formes de couronne. Ceci a été réalisé par une étude descriptive de la croissance de deux clones, PB 5/51 et RRIM 600, reconnus par lensemble des auteurs comme respectivement caractéristiques des phénotypes résistant et sensible à la casse au vent. Les résultats rapportés ici concernent cette premièrétape. Les 2 clones se différencient par de nombreux paramètres.

Dans une deuxième étape, afin de ne reternir comme critères de sélection que les plus significatifs, on a établi une hiérarchie entre ces paramètres en utilisant les méthodes 
de l'analyse multivariable. On a également vérifié que les critères retenus pouvaient être évalués de façon précoce (avant 2.5 ans). Cette deuxième étape du travail qui permet de proposer des critères de sélection précoce sera publiée prochainement.

\section{Matériels et méthodes}

Une des caractéristiques de la morphogénèse de l'hévéa est sa croissance par poussées successives (Dubois, 1962). L'élément de base de cette croissance rythmique est lUnité de Croissance, U.C. (Halle \& Martin, 1968). Le rythme de croissance est d'origine endogène mais varie selon les clones et les conditions environnementales. L'U.C. mise en place au cours d'une phase de croissance est composée de deux zones morphologiquement distinctes :

- la zone inférieure, qui est celle des bourgeons d'écaille préformés dans le bourgeon terminal de ['U.C. précédente,

- la zone supérieure, qui est celle des bourgeons de feuille néoformés lors du développement de l'U.C.

Ces 2 types de bourgeons ont des devenirs spécialisés : les rameaux végétatifs ne se développent qu'à partir des bourgeons de feuille, les bourgeons d'écailles sont à l'origine des axes florifères.

Dans la classification des modèles architecturaux des arbres tropicaux (Halı: \& Oldeman, 1970), lhévéa est conforme au modèle de RauH. Il présente un tronc monopode à croissance rythmique conférant aux branches une disposition verticillée ou subverticitléc, des branches orthotropes à croissance rythmique, une floraison latérale sur branches ou tronc: les méristèmes ont un fonctionnement indéfini permettant d'édifier des branches monopodes.

Les arbres utilisés dans cette étude sont des clones de greffe, clone pour la partie aérienne, tous greffés sur la même famille de porte-greffe (descendance maternelle) qui sert de partic racinaire. Ce type de matériel végétal est le plus largement utilisé dans les plantations incustriclles en raison de ses meilleures caractéristiques d'homogénéité comparé au matéricl issu de semences.

Trente-huit individus du clone PB $5 / 51$ et 40 du clone RRIM 600 ont été étudiés dans un dispositif installé au sud-ouest de la Côte-d'lvoire. La densité de plantation était de 510 arbres par hectares $(7 \mathrm{~m} \times 2,8 \mathrm{~m})$.

Sur chaque incividu 4 notations successives de l'état de dévcloppement ont été réalisées, la première à 18 mois et les 3 suivantes à un an d'intervalle. Les arbres ont été abattus pour effectuer la dernière notation.

Les connaissances de base de la morphogénèse de lhévéa ont permis de représenter chaque individu sous forme d'un diagramme (fig. 2) en utilisant les 4 notations successives du développement. Chaque notation a consisté à repérer :

- les U.C. existantes et leurs longueurs,

- les bourgeons de feuille ayant donné naissance à un axe végétatif (numéroté dans lordre de la phyllotaxie),

- Les mortalités des bourgeons terminaux. 


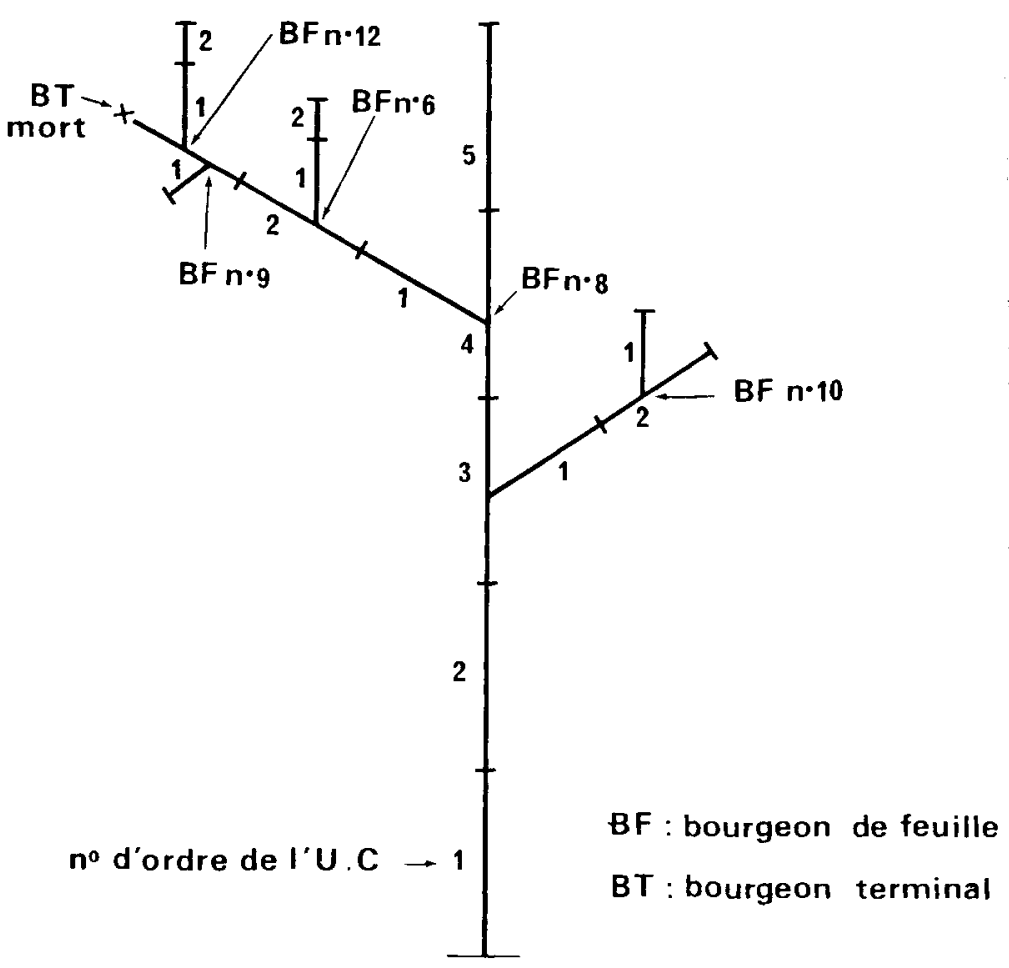

FIG. 2

Diagramme de ramification.

Branching pattern.

Ces notations ont été réalisées sur les axes primaires, secondaires et tertiaires. L.e système de codage utilisé permet de décrire un individu par l'ensemble de ses unités de croissance et leurs relations de succession dans le temps (année de mise en place) et dans l'espace (position dans larbre).

Un relevé exhaustif des angles d'insertion des branches sur l'axe 1 et les axes 2 a, d'autre part, été réalisé sur un autre échantillon composé de 5 arbres de chaque clone PB 5/51 et RRIM 600 âgés de 5 ans.

A partir des données descriptives issues des diagrammes de développement nous avons défini un ensemble de variables permettant de représenter l'édification et les caractéristiques des axes 1, 2, 3, et le fonctionnement des bourgeons de feuille de laxe primaire (ou secondaire) pour donner les axes secondaires (ou tertiaires). 


\section{Résultats}

\subsection{Longueurs des U.C.}

Le tableau 1 donne les longueurs moyennes des U.C. des axes 1,2 et 3 pour chacun des clones. Quel que soit laxe, les U.C. de PB 5/51 sont significativement plus longues que celles de RRIM 600. Dans chaque clone la taille des U.C. décroît des axes 1 aux axes 3 .

\section{Tableau 1}

Longueurs moyennes des U.C. (en $\mathrm{cm}$ ).

Mean lengths of grow'th whits (in $\mathrm{cm}$ ).

\begin{tabular}{ccc|c|c}
\hline & & & PB 5/51 & RRIM 600 \\
\hline Axe 1 & $\ldots$ & 55,12 & 52,08 \\
Axc 2 & $\ldots$ & $\ldots$ & 42,30 & 39,30 \\
Axe 3 & $\ldots$ & 38,90 & 35,20 \\
\hline
\end{tabular}

Les longueurs moyennes des U.C. daxe 1 ont également été comparées entre arbres d'un même clone. Les valeurs du $F$ de lanalyse de variance $(25,8$ pour RRIM 600 contre 1,2 pour $\mathrm{PB} 5 / 51$ ) indiquent l'existence d'une forte hétérogénéité intraclonale pour ce caractère dans le clone RRIM 600.

La courbe de la figure 3 représente pour chaque clone la variation de la longueur moyenne des U.C. d'axe 1 en fonction de leur position sur cet axe (numéro d'ordre). On retrouve pour chaque clone des maximums associés aux mêmes numéros d'U.C. $(6,11$ et 14). Cette coïncidence ne peut être attribuée à des facteurs climatiques, car on a montré (HoFmanN, 1981) que les U.C. 11 et 14 ne sont pas mises en place simultanément dans les 2 clones. Il existe donc vraisemblablement un rythme endogène influençant la longueur des U.C. successives sur l'axe 1.

\subsection{Nombre d'Unités de Croissance sur laxe 1}

Le nombre total d'U.C. d'axe 1 est plus important pour RRIM 600 que pour PB 5/51 (16,4 contre 11,2$)$ et la variabilité entre arbres est 3 fois plus forte chez RRIM 600.

I.e tableau 2 donne le nombre d'U.C. d’axe 1 mises en place au cours des périodes de croissance observées. De 60 à 70 p. 100 du nombre total d'U.C. d’axe 1 sont mises en place avant 18 mois. La différence entre les 2 clones persiste quelle que soit la période de croissance envisagée, mais est maximum pendant cette première phase. 
Les corrélations entre le nombre d'U.C. d'axe 1 mises en place au cours des différentes périodes d’observation (tabl. 3) soulignent cette particularité du fonctionnement avant 18 mois. On observe des corrélations négatives ou nulles entre les périodes de croissance codées 0 et $1^{(2)}$ puis des corrélations positives dans les périodes suivantes.

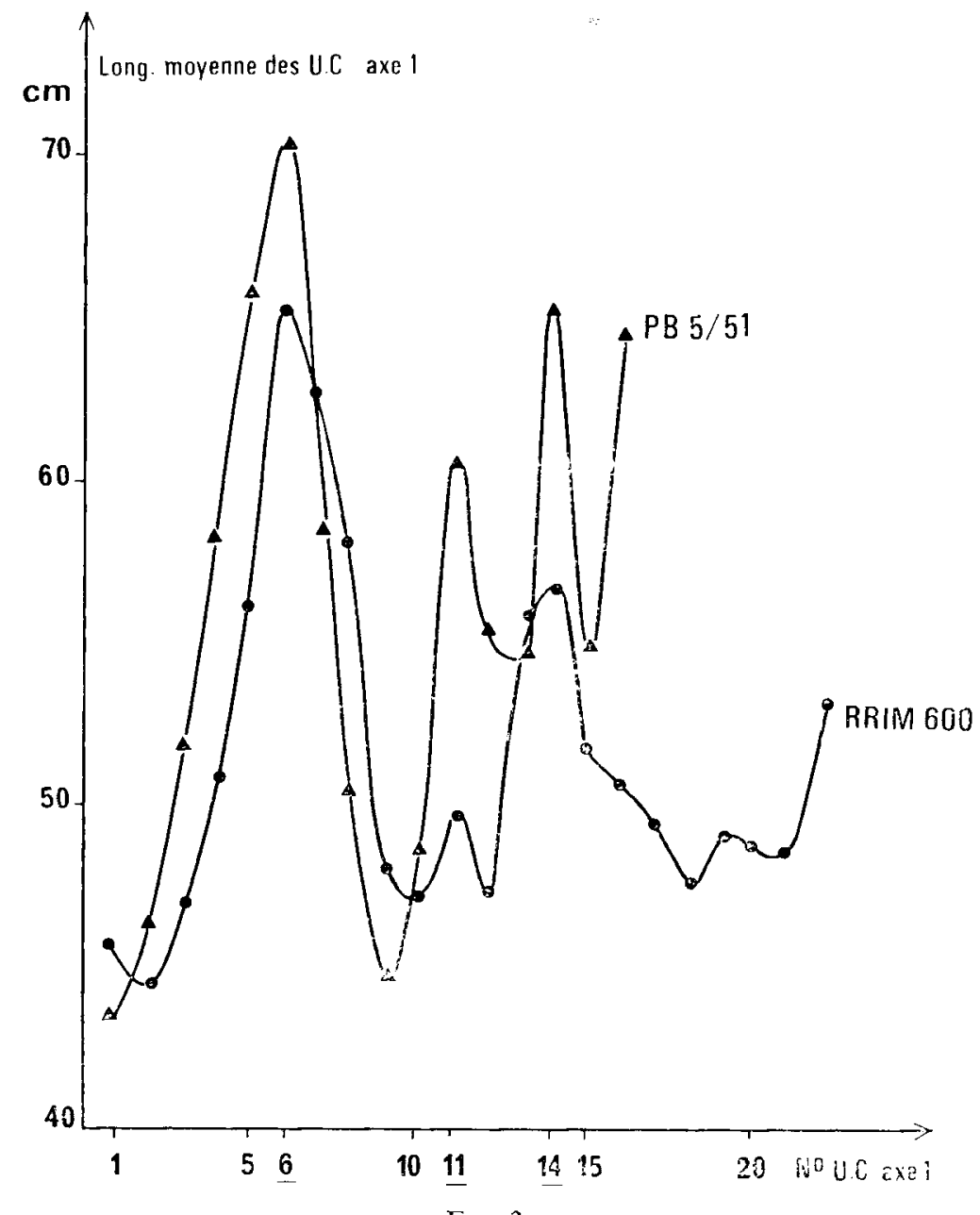

Fig. 3

Longueurs moyennes des U.C. selon lear position sur l'axe 1.

Mean lengths of growth units according to their position on the primary axis.

(2) Dans tous les lableaux les codes $0,1,2,3$ correspondent respectivement aux périodes de croissance 0 à 18 mois, 18 mois à 2,5 ans, $2,5 \grave{a} 3,5$ ans et $3,5 \grave{a ̀ ~} 4,5$ ans. 
TABLEAU 2

Nombre dU.C. daxe 1.

Number of primary axis growth wits.

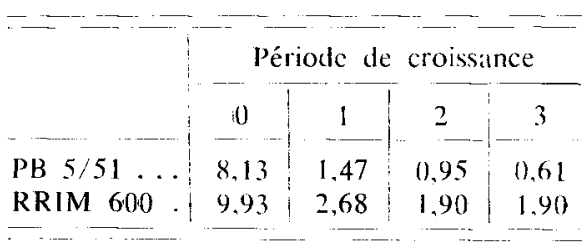

Les codes $0,1,2,3$ correspondent aux pérjodes de croissance 0 à 18 mois, 18 mois à 2,5 ans, 2,5 à 3,5 ans et 3,5 à 4,5 ans.

The codes $0,1,2,3$ correspond to the periods of grolith from 0 to 18 months, 18 months io 2.5 years, 2.5 to 3.5 years, 3.5 to 4.5 years.

\section{TABLFAU 3}

Corrélations entre les nombres d'U.C. mises en place

an cours des périodes successives de croissance.

Correlations between the number of growth units

appeared during successive growth periods.

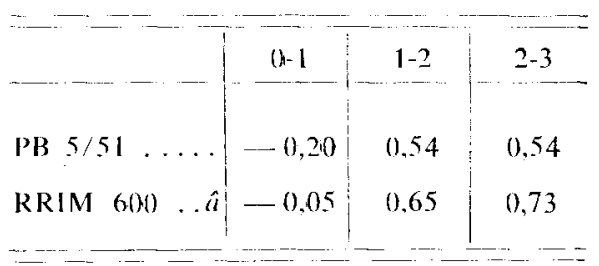

\subsection{Péremnité du fonctionnement du méristème terminal d'axe I}

La probabilité d’arrêt de fonctionnement du méristème terminal d’axe 1 (tabl. 4) peut être estimée d’après la période de mise en place de l'U.C. terminale sur chaque individu.

Les valeurs observées infirment pour laxe 1 la notion de croissance indéfinie postulée dans le modèle de RauH. En effet dès 18 mois, 42 p. 100 des arbres du clone PB 5/51 et 27 p. 100 de ceux du clone RRIM 600 n'édifient plus de nouvelles U.C. d'axe 1 ; à 3,5 ans ces fréquences atteignent respectivement 76 p. 100 et 50 p. 100 . Ce phénomène napparaît pas avec la même fréquence dans les 2 clones; il est beaucoup plus marqué chez PB 5/51 et semble stable sur les 3 intervalles de croissance où il a pu être estimé.

TABLEAU 4

Probabilité d'arrêt de fonctionnement du méristème terminal d'axe 1.

Growth stopping probability of the primary axis terminal meristem.

\begin{tabular}{|c|c|c|c|}
\hline & \multicolumn{3}{|c|}{ Période de croissance } \\
\hline & 0 & 1 & 2 \\
\hline PB $5 / 51$ & 0,42 & 0,41 & 0,31 \\
\hline RRIM 600 & 0,27 & 0,17 & 0,18 \\
\hline
\end{tabular}


Cet arrêt de fonctionnement du bourgeon terminal, plus fréquent chez. PB $5 / 51$, n'est pas associé à une différence de hauteur du tronc observable en plantation. Ceci implique donc que les bourgeons axillaires de I'U.C. terminale prennent rapidement le relais de laxe 1 pour la formation du tronc. Deux types de fonctionnement de laxe 1 semblent donc exister, l'un à croissance "définie» associé à un phénomène de relais par des axes secondaires, lautre à croissance «indéfinie».

L'existence du phénomène de relais orthotrope postulée jei a pu être montrée anatomiquement sur un autre clone, PB 86 (HOFMANN, 1981).

\subsection{Présence de ramifications}

Le démaragge de ramifications sur une U.C. de niveau $N$ se produit généralement pendant le développenent de IU.C. $N+1$ (ramification proleptique). Toutes les U.C. ne sont cependant pas ranifiées.

\subsubsection{Axe 1}

La fréquence dindividus dont toutes les U.C. daxe 1 sont ramifiés al est de 74 p. 100 pour PB $5 / 51$ et de 20 p. 100 pour RRIM 600.

La fréquence dU.C. daxe 1 ramifiées est de 91,6 p. 100 chez PB $5 / 51$ et de 76,6 p. 100 chez RRIM 600 ; ces différences sont significatives. Ceci correspond ì un nombre moyen dU.C. non ramifiées par individu de 1,3 chez PB $5 / 51$ contre 2.9 chez RRIM 600. La différence la plus marquée entre les 2 clones est associée à la période de croissance située avant 18 mois. Les U.C. non ramifiées ne correspondent pas à des positions spécifiques sur l'axe 1, mais peuvent être considérées comme réparties au hasard.

\subsubsection{Axic ?}

Le même critère a été éludié pour les U.C. daxe 2. Les 2 clones se comportent de la même façon, mais la fréquence d'U.C. ramifiées est beaucoup plus faible que sur laxe $1: 36,6$ p. 100 pour PB $5 / 51$ at 32,2 p. 100 pour RRIM 600 .

La courbe (fig. 4) des fréquences dU.C. daxe 2 ramifiées en fonction du numéro d'ordre sur laxe 2 montre que les valeurs ci-dessus recouvrent des situations très différentes selon le numéro d'U.C. ¿"axe 2. La variation esi cependant la même dans les 2 clones.

\subsection{Position des ramifications à l'intérieur de l'U.C.}

La répartition des branches à lintérieur de I’U.C. est représentée par les courbes de fréquence de fonctionnement cies bourgeons de feuille repérés dans lordre de la phyllotaxie.

Sur les U.C. daxe 1 (fig. 5), le clone RRIM 600 présente un maximum de ramifications au niveau des bourgeons de numéros 5 , 6 et 7 et une diminution rapide des

(3) A partir de lU.C. 4. car toute ramification démarrant sur les U.C. 1 ì 3 est systématiquement éliminée pour disposer ensuite d'un panneau de saignée sans branches. 
fréquences associées aux numéros de bourgeon suivants. PB $5 / 51$ présente un maximum de ramifications réparti sur les bourgeons 4 à 8 et la distribution est plus étalée vers les numéros de bourgcons plus élevés.

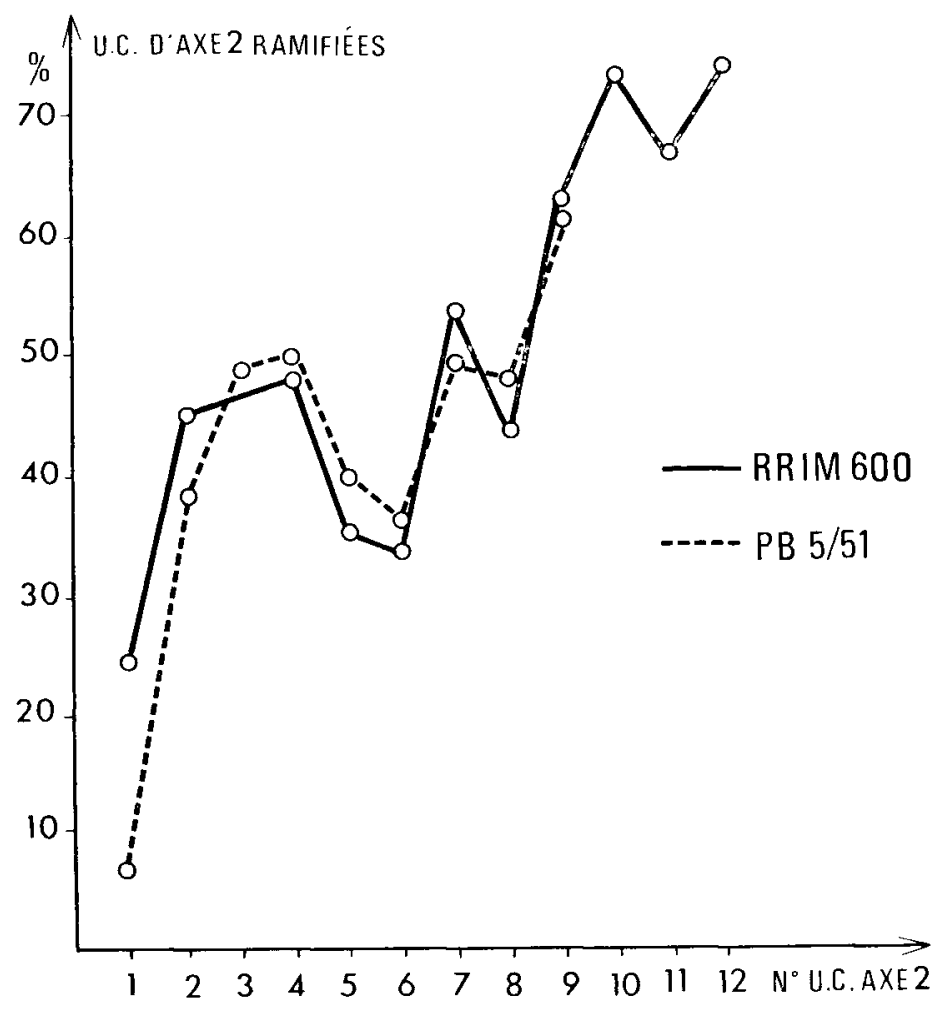

FIG. 4

Fréquence des U.C. d'axe 2 ramifiées.

Frequency of ramified secondary axis growth units.

Pour les U.C. d'axe 2 (fig. 6), les 2 clones ont un comportement identique; la zone de fonctionnement maximum se situe au niveau des bourgeons 3 à 7 .

\subsection{Nombre de branches par U.C. ramifiée}

Le tableau 5 donne pour les différentes périodes de croissance le nombre moyen de branches par U.C. d'axe 1 ramifiée.

Le nombre de branches de PB 5/51 est supérieur à celui de RRIM 600 pour les différentes périodes de croissance considérées ; ce paramètre est assez stable au cours du temps et on n'observe pas de différences significatives entre arbres d'un même clone. 


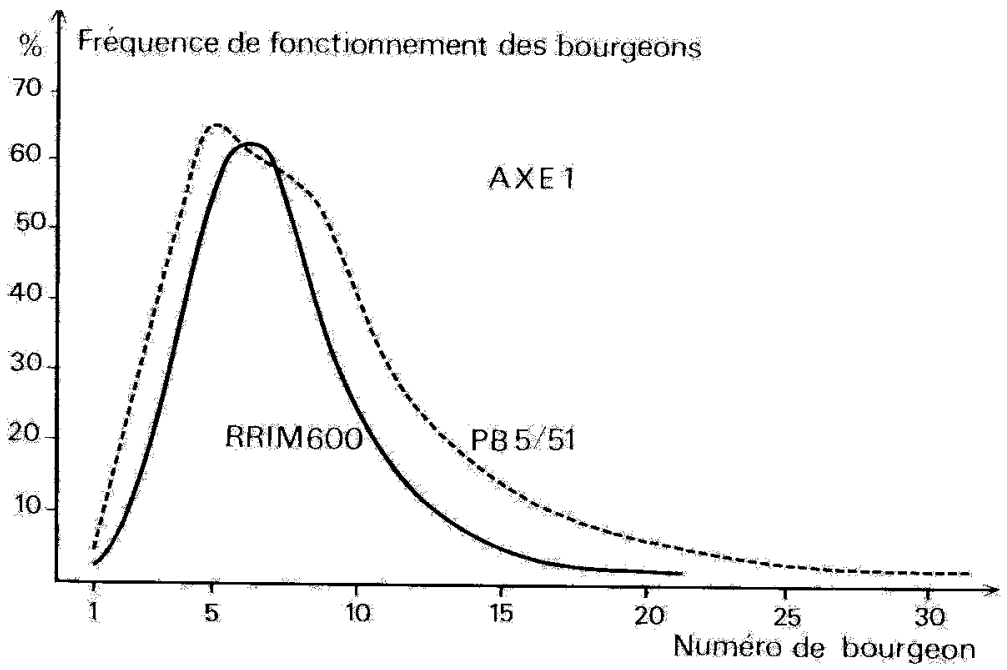

FIG. 5

Fréquence de fonctionnement des bourgeons de feuilles d'axe 1.

Functioning frequency of primary axis leaf buds.

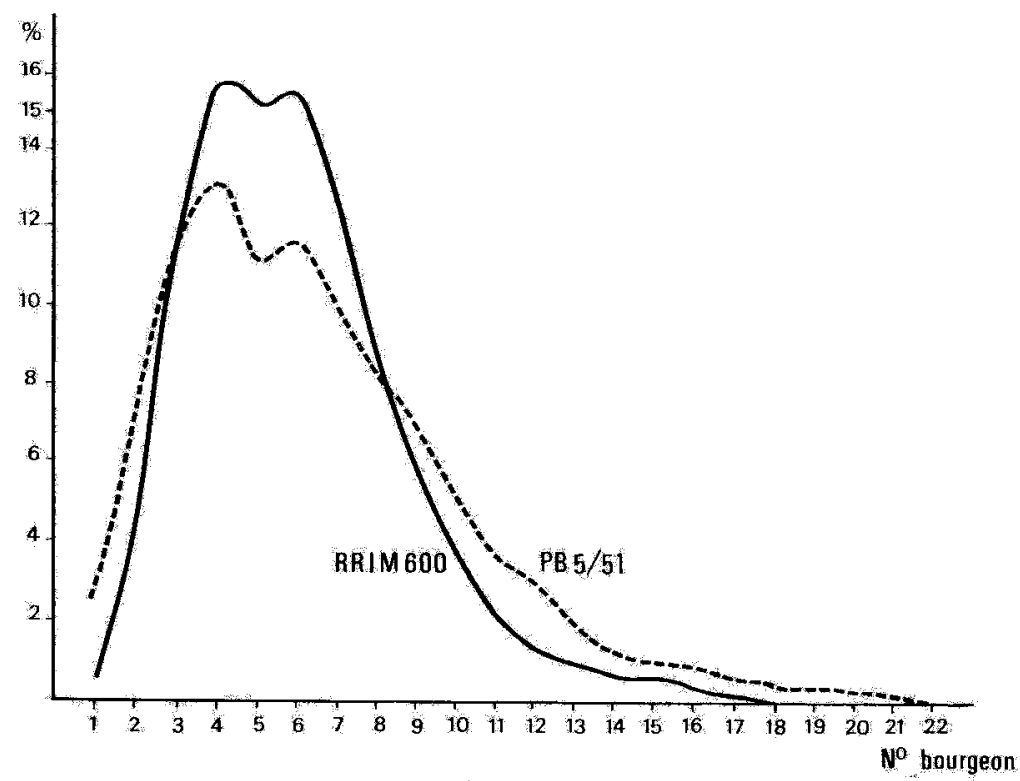

FiG. 6

Fréquence de fonctionnement des bourgeons d'axe 2.

Functioning frequency of secondary axis leaf buds. 
Cette différence entre clones reste vraic si l'on considère le nombre de ramifications en fonction des numéros d'U.C. d'axe 1 (fig. 7); la courbe de PB 5/51 reste toujours au-dessus de celle de RRIM 600.

\section{Tabienu 5}

Nombre de branches par U.C. ramifiée.

Number of branches per ramified growth unit.

\begin{tabular}{cc|c|c|c|c}
\hline & \multicolumn{3}{|c|}{ Période de croissance } \\
\hline & & 0 & 1 & 2 & 3 \\
\hline & & & & \\
\hline PB $5 / 51$ & $\ldots a \hat{a}$ & 6,2 & 6,7 & 5,1 & 6,6 \\
RRIM 600 & 3,6 & 4,0 & 4,1 & 5,9 \\
\hline
\end{tabular}

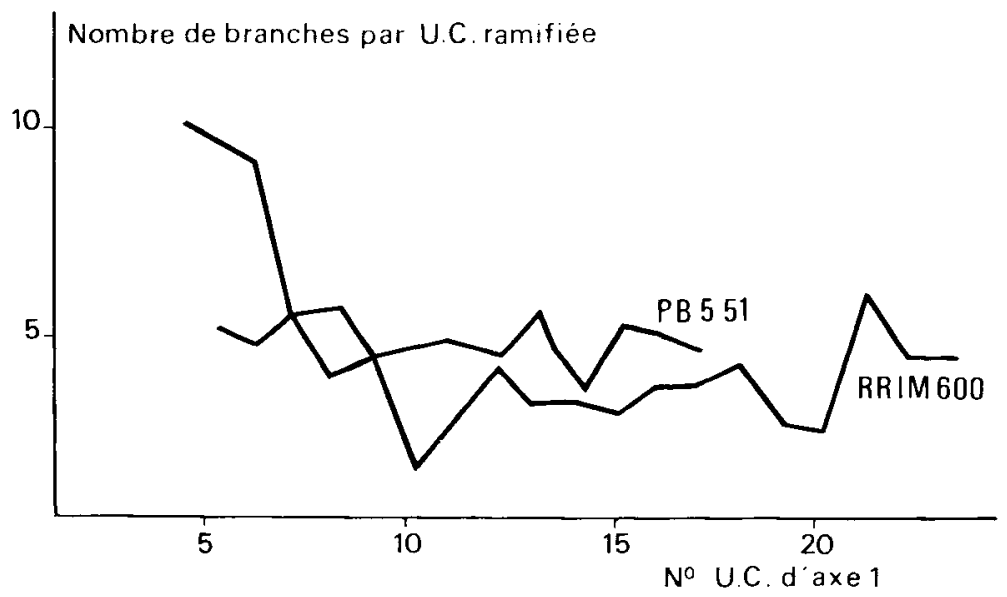

Fig. 7

Nombre de branches par U.C. ramifiée selon le niveau d'U.C. d'axe 1.

Number of branches per ramified growth unit according to their position on the primary axis.

Il en est de même pour les branches (axes 3) portées par les U.C. d'axe 2 (fig. 8). La différence entre les 2 clones est cependant moins marquée : PB 5/51 porte en moyenne 3,1 branches par U.C. d'axe 2 ramifiée et RRIM 600 2,8. 
Nbre de branches / U.C. ramifiée

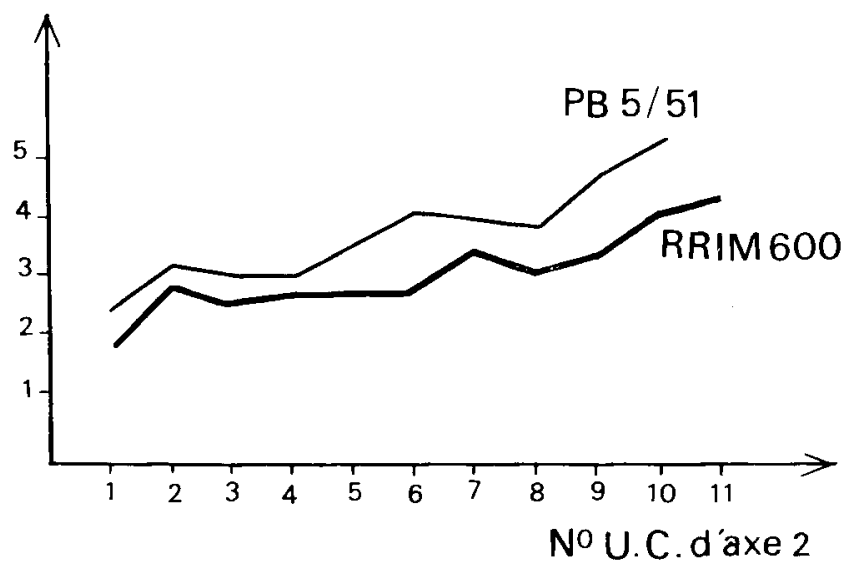

FIG. 8

Nombre d'axes 3 par U.C. ramifiée selon le numéro d'U.C. d'axe 2.

Number of tertiary axis per ramified growth unit according to their position on secondary axis.

\subsection{Mortalité des branches}

La notation de mortalité des bourgeons terminaux permet de classer les ramifications en branches vivantes et branches mortes. Cette mortalité se traduit par un dessèchement de la branche qui concluit à un élagage naturel.

Sur la fïgure 9 la mortalité est exprimée pour chaque clone et pour chaque intervalle de croissance par la distance entre les points correspondant au nombre total des branches d'une part et au nombre de branches vivantes par U.C. ramifiée d'autre part.

PB $5 / 51$

RRIM 600

Nbre de branches par U.C ramifiée
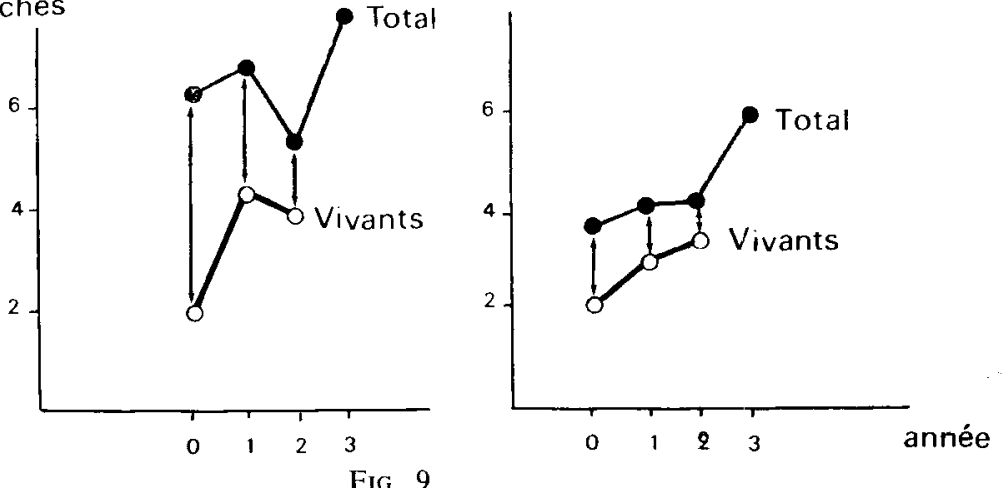

Mortalité des branches.

Branching mortality. 
Ce phénomène de mortalité est beaucoup p'us marqué chez le clone PB 5/51 que sur RRIM 600. Il est quantitativement important puisque dans la période de croissance $0-18$ mois, il concerne respectivement 70 p. 100 et 43 p. 100 des ratmifications de PB $5 / 51$ et RRIM 600).

\subsection{Longueur des branches}

La longueur des branches a été exprimée en nombre dUU.C.: te lableaulu 6 donne les résultats pour les branches vivantes et les branches mortes.

Les différences entre les 2 clones sont significatives pour les 2 calégories de branches; les branches mortes correspondent à des branches courtes clans les 2 clones : 95 p. 100 de celles-ci sont composées de 2 ou 3 U.C. au plus. Les branches vivantes de RRIM 600 sont plus longues que celles de PB 5/51.
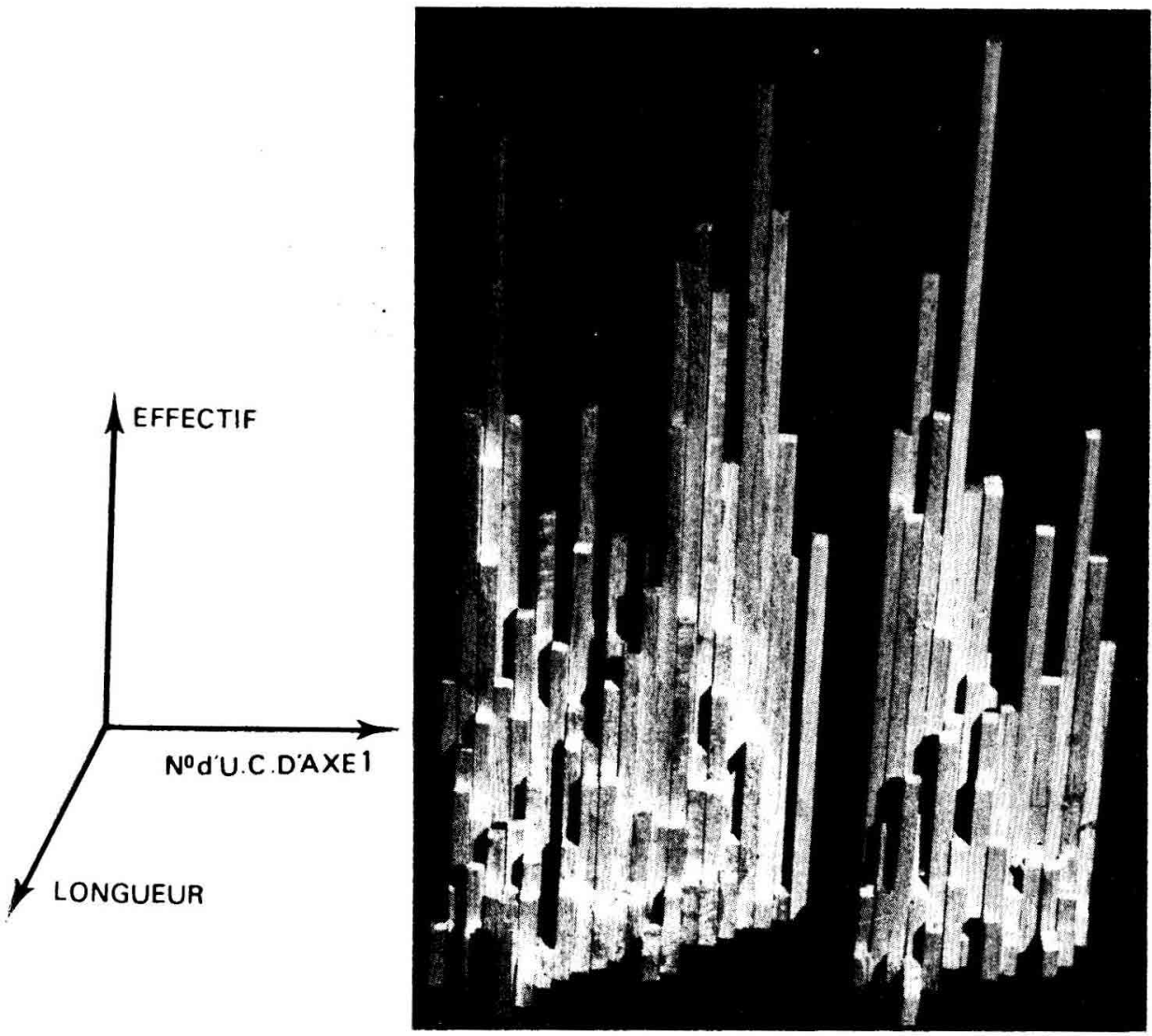

RRIM 600

$\mathrm{PB} \quad 5 / 51$

FIG. 10

Histogrammes des longueurs de branches selon les numéros d'U.C. d'axe 1 . Histograms of branch lengths according to the primary axis growth unit number. 
La même tendance existe pour la longueur des branches portées par les U.C. daxe $2(2,4$ pour RRIM 600 et 2,2 pour PB $5 / 51)$.

Lhistogramme (fig. 10) des longueurs des axes 2 en fonction de leurs positions sur l'axe 1 montre que la différence de répartition des longueurs des branches est essentielement associée à la différence de développement de l'axe 1. On observe dans les 2 clones, une zone de maximums de longueur des branches au niveau des U.C. de numéros 6,7 et 8 ; il existe de plus chez RRIM 600, au niveau des U.C. 24 et 25 , une deuxième zone de fréquences élevées de branches longues.

\subsection{Angles d'insertion des branches}

Le tabieau 7 présente les moyennes et les écart-types des angles d'insertion des branches sur laxe 1 et laxe 2.

\section{TABLeau 6}

Longueurs des branches virantes et mortes (en nombre dU.C.).

Lengths of living and dead branches (in number of grow'th units).

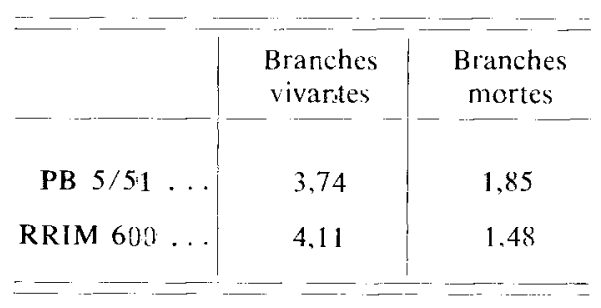

\section{Tableau 7}

Moyennes et ćcart-types des angles d'insertion des branches sur les axes $l$ et 2.

Mealls and standard deviations of branching angles on primary and secondary axis.

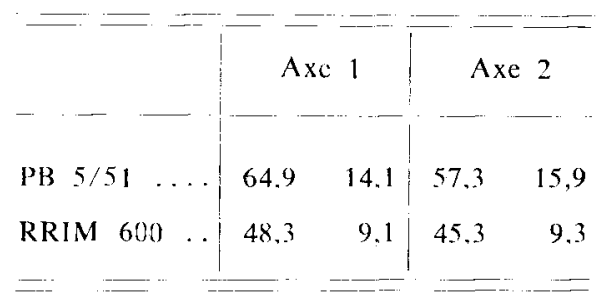

La proximité des valeurs de moyenne et variance et la ressemblance des distributions (non présentées ici), entre axe 1 et 2 sont un bon indice de la stabilité de ce paramètre tout au long de la vie de l'arbre.

La différence entre clones est évidente : PB 5/51 présente des angles d'insertion nettement plus ouverts que ceux de RRIM 600.

\section{Discussion}

L'ensemble des paramètres étudiés présente des différences significatives entre les. 2 clones résistant et sensible à la casse au vent. Parmi ceux-ci, certains paraissent particulièrement intéressants. 
Le fonctionnement théoriquement indéfini de l'axe 1 est loin d’être la règle générale. On peut considérer que coexistent dans les 2 clones, mais avec des fréquences différentes, 2 types de fonctionnement de laxe 1 : celui à croissance limitée associé à la présence de relais orthotropes par un ou des axes secondaires. et celui à croissance illimitée.

La différence de ramification entre les 2 clones n'est pas due au nombre total de branches mais à leurs caractéristiques de répartition à l'intéricur de rU.C., d'angles d'insertion, de mortalité et de longueurs.

Pour l'ensemble des critères observés les différences sont plus marquées pour ceux concernant la mise en place des axes 1 et 2 . Les différences entre les 2 clones s'estompent au niveau des axes 3 .

Bien que lon n’ait pas présenté ici les résultats de variabilité intraclone associéc à chacun des critères, on doit signaler que de façon quasi systématique, la variabilité observée clans le clone RRIM 600 est nettement plus forte que celle du clone PB $5 / 51$. La morphogénèse du clone PB $5 / 51$ semble plus étroitement définie que celle de RRIM 600.

Lorsque lévolution des variables en fonction du temps (périodes de croissance) ou de la position dans l'arbre (numéro d'U.C.) a été étudiée, les valeurs observées sont stables, ou bien évoluent de façon régulière. Ceci permet de penser que les variables définies à partir des données descriptives ont un sens du point de vue de la morphogénèse.

On peut proposer une description schématique comparative des 2 clones :

PB 5/51 est caractérisé par :

- un développement limité de l'axe 1, en nombre d'U.C. et dans le temps,

- une ramification installée régulièrement le long de l'axe 1, et ćtalće à l'intéricur de I'U.C.,

- une mortalité importante des branches,

- un nombre de branches par U.C. ramifiée important,

- des branches courtes,

- des angles d'insertion des branches ouverts.

RRIM 600 est caractérisé par :

- un développement plus important de l'axe 1 ,

- la présence d'U.C. non ramifiées, distribuées au hasard sur laxe 1,

I'U.C.,

- une ramification moins nombreuse et plus étroitement répartie à lintérieur de

- des branches longues,

- des angles d'insertion des branches plus fermés.

La mise en évidence sur des critères de morphogénèse, de différences entre deux clones résistant et sensible à la casse au vent est une première étape vers la définition de critères de sélection précoce. L'étape suivante consiste à choisir un nombre minimum de caractères exprimant au mieux et à un stade juvénile les différences existint entre les deux clones. Ceci a été réalisé en utilisant une approche multivariable qui permet de hiérarchiser les différences observées, en tenant compte en particulier de la variabilité intraclonale. Les variables retenues peuvent être directement ulilisées comme critères de sélection précoce. 


\section{Summary \\ Morphogenetic study of the crownshape of wind-susceptihle. and resistant clones of Hevea brasiliensis.}

In Hevea brasiliensis, there is a relationship between crownshape and wind susceptibility or resistance. It can be used to look for early selection criteria. The growth of a resistant (PB 5/51) and a susceptible (RRIM 600) clone was studied. A set of variables which represents the edification and the characteristics of primary, secondary and tertiary axis was defined. Between-clone differences especially concern the growth stopping on the primary axis, the regularity of branching, the length, number and mortality of branches, the branching angles. Within-clone-variability is higher on the susceptible clone (RRIM 600) for nearly all the traits. The availability of easily measurable variables which display differences between wind-susceptible and resistant clones, is a first step towad the derinition of useful early selection criteria.

\section{Références bibliographiques}

ANON, 1974. Wind damage and pruning. Planters Bull., R.R.I.M., 132, 77-97.

Combr, J.Cl., du Plessix C.J., 1974. Etude du développement morphologique de la couronne de Helea brasiliensis (Müll.-Arg.) (Euphorbiacécs-Crotonoïdées). Ann. Sci. For., 31 (4), 207-228.

Dubors, 1962. Contribution à l'étude de la croissance par poussées successives chez Hevea brasiliensis (Müll.-Arg.). Agricultura, Louvain, 125-149.

Halle F., Oldeman R.A.A., 1970. Essai sur larchitecture ef la dynamique de croissance des arbres tropicallx. Monographie n" 6, Masson, Paris, 178 p.

Halle F., Martin R., 1968. Etude de la croissance rythmique chez l'Hévéa (Hevea brasiliensis, Müll.-Arg.) (Euphorbiacées-Crotonoüdes). Adansonia Ser., 2 (8), 475-503.

HoFmann J.P., 1981. Recherche de critères de sélection pour la résistance à la casse au vent chez. Hevea brasiliensis : étude morphogénétique de la couronne et modèle de simulation de la croissance. Thèse de doctorat ès-Sciences Naturelles, Université Paris XI,

224 p. + annexe.

Nicolas D., Hormann J.P., 1977. - Etude de la présence de bois de tension en relation avec le phénomène de la casse au vent chez Hevea brasiliensis. Rapport Inst. Recherche Caoutchouc (I.R.C.A.).

Nicol_as D., Magnin E., Hol:mann J.P., 1979. Contribution à l'étude des relations entre certaines caractéristiques du bois et la sensibilité à la casse chez des clones d'Hevea brasiliensis. Rapport Inst. Recherche Caoutchouc (I.R.C.A.).

OSTENDORFF IR. F.W., 1931. Een ongewenscht verta ingstype (an undesirable type of branching). De Bergeucultures 1931, 31 (1), 825.

Wycherley P.R., 1969. Breeding of Hevea. J. Rubb. Res. Inst. Malaya, 21 (1), 38-55.

Yoon, 1976. An approach to modify branching habits. Its effects and potentials. Proc R.R.I.M.'s Planters Conf., 143-158. 